\title{
Design of an Enterobacteriaceae Pan-genome Microarray Chip
}

\section{Lukjancenko, Oksana; Ussery, David}

\section{Published in:}

CSBio 2010

Publication date:

2010

Document Version

Publisher's PDF, also known as Version of record

Link back to DTU Orbit

Citation (APA):

Lukjancenko, O., \& Ussery, D. (2010). Design of an Enterobacteriaceae Pan-genome Microarray Chip. In CSBio 2010 (Vol. 115, pp. 174-189). Springer.

\section{General rights}

Copyright and moral rights for the publications made accessible in the public portal are retained by the authors and/or other copyright owners and it is a condition of accessing publications that users recognise and abide by the legal requirements associated with these rights.

- Users may download and print one copy of any publication from the public portal for the purpose of private study or research.

- You may not further distribute the material or use it for any profit-making activity or commercial gain

- You may freely distribute the URL identifying the publication in the public portal

If you believe that this document breaches copyright please contact us providing details, and we will remove access to the work immediately and investigate your claim 


\title{
Design of an Enterobacteriaceae Pan-Genome Microarray Chip
}

\author{
Oksana Lukjancenko and David W. Ussery \\ Center for Biological Sequence Analysis, Department of Systems Biology, \\ The Technical University of Denmark, 2800 Kongens Lyngby, Denmark
}

\begin{abstract}
Microarrays are a common method for evaluating genomic content of bacterial species and comparing unsequenced bacterial genomes. This technology allows for quick scans of characteristic genes and chromosomal regions, and to search for indications of horizontal transfer. A high-density microarray chip has been designed, using 116 Enterobacteriaceae genome sequences, taking into account the enteric pan-genome. Probes for the microarray were checked in silico and performance of the chip, based on experimental strains from four different genera, demonstrate a relatively high ability to distinguish those strains on genus, species, and pathotype/serovar levels. Additionally, the microarray performed well when investigating which genes were found in a given strain of interest. The Enterobacteriaceae pan-genome microarray, based on 116 genomes, provides a valuable tool for determination of the genetic makeup of unknown strains within this bacterial family and can introduce insights into phylogenetic relationships.
\end{abstract}

Keywords: Enterobacteriaceae, Pan-genome, DNA microarray analysis, gene, Escherichia coli.

\section{Introduction}

The risk of dying from disease caused by a bacterial infection is greater than that associated with any other type of disease, including cancer or heart attacks $[1,2]$. Epidemic infectious diseases are the most serious causes of mortality and morbidity worldwide, more than all other diseases combined. Infections contribute to significant economic loss in most parts of the world, including first world countries that have high income and developed surveillance and control systems [3, 4]. Every year thousands of people are infected by bacterial pathogens, most of which are transmitted through food [5]. The outcome from food-borne human infections can range from mild self-limiting diarrhea to severe illness that requires hospitalization. In rare cases, food-borne illnesses are even fatal [5, 6]. Enteric bacteria, particularly Salmonella enterica subsp. enterica, are among the leading food-borne pathogens [6, 7]. In light of this, the detailed and rapid investigation of enteric pathogens is essential in modern epidemiology and clinical diagnostics.

Enterobacteriaceae are pervasive. They are widespread in the environment, existing in water, soil, food, and plants, as well as in the normal intestinal flora of many animals and humans [8-12]. Pathogens within this group have developed a diversity 
of strategies to overcome protective host barriers in order to invade the host, resist innate immune response, multiply in specific and normally sterile body sites, and damage cells in order to establish and maintain a successful infection [13, 14]. Genera within Enterobacteriaceae family are of interest, as well, because of problems from food spoilage and for that reason are of considerable economic importance [15].

Bacterial genomes vary in size, even among the strains of the same species. Bacterial species can be characterized by its pan-genome. As defined by Tettelin et al., the microbial pan-genome is a complete collection of various genes located within populations at a particular taxonomic level, commonly within a species. The pan-genome concept can of course be expanded to higher levels, such as genus or even a bacterial family. The pan-genome includes a core-genome, which is a minor fraction of the entire gene pool that is shared between all the given strains. Furthermore, there is a much larger, dispensable portion of bacterial genes, that are missing in one or more strains. Also there are some genes that appear to be unique to each strain $[16,17]$. Strain-specific genes can, even among a particular species, make up a notably large portion of the pan-genome [18].

Many methods have been developed for characterizing genetic variation. Use of DNA microarrays is becoming a standard procedure for evaluating genotyping - that is, looking at the genetic content of a bacterial species. The price for microarrays used for genotyping was historically expensive, but now is becoming competitive with the cost of other commonly used typing methods, such as previously widely used multilocus sequence typing (MLST). Moreover, it is becoming increasingly popular, quick, and cost-effective to define the presence and absence of each of the assigned genes in the pan-genome of a species. Thus, microarrays, imprinted with all the genes from species' pan-genome can be used to compare and characterize the genomic content of unknown bacterial isolates and to achieve accurate typing information, that can be useful in epidemiological investigations and clinical diagnostics [1, 19]. For instance, array comparative genomic hybridization $(\mathrm{aCGH})$ is frequently used in human cancer studies to genotype cell lines by determination of gene loss and copy number variations [20] or to detect single nucleotide polymorphisms at target loci [21]. Additionally, microarrays have been widely used in human screenings for the determination and genotyping of bacterial species. Microarrays have changed considerably since they were first introduced. Early microarrays for the E. coli genome consisted of long fragments of chromosomal DNA ( 1000 to 2000 base-pairs), attached to a microscope slide. Later, Affymetrix made an array covering the entire E. coli K-12 genome using a set of 10 to 15 probes (synthetic 25mers) for each gene [22], followed shortly by an array which contained 4 E. coli genomes [23, 24]. Custom-designed NimbleGen chips have been made including 7 and then 32 E. coli genomes [25, 26].

This study describes the design and use of a high-density oligonucleotide microarray covering the pan-genome of 116 genomes within the Enterobacteriaceae family. Probes are designed to distinguish among organisms at the level of genera, species, and even single strains. Moreover, probes for determination of particular gene families, comprising Enterobacteriaceae pan-genome, are defined. The performance of this microarray is evaluated both in silico and experimentally. Its utility is illustrated for the hybridization of genomic DNA in order to compare uncharacterized isolates which have not been sequenced with the 116 known, sequenced strains. A microarray chip approximating the complete pan-genome of Enterobacteriaceae 
provides optimal sensitivity to characterize isolates. Gene family microarray analysis is useful for medical and environmental diagnoses and will provide an alternative to costly genome libraries, as well as to the sequencing of environmental samples.

\section{Materials and Methods}

\subsection{Bacterial Strains}

In this study, one hundred and twelve complete Enterobacteriaceae genome sequences and four in progress, which were publically available in GenBank database at the time of analysis (February, 2010), were used for custom microarray design. An overview of the used strains is shown in Table 1 and the complete collection of the strains is described in supplementary Table $\mathrm{S} 1^{1}$.

Table 1. Enterobacteriaceae genera used in the design of the microarray chip

\begin{tabular}{lclc}
\hline Genus & Number of strains & Genus & Number of strains \\
\hline Buchnera & 6 & Photorhabdus & 2 \\
Citrobacter & 3 & Salmonella & 18 \\
Cronobacter & 2 & Serratia & 1 \\
Dickeya & 3 & Shigella & 8 \\
Edwardsiella & 2 & Sodalis & 1 \\
Enterobacter & 2 & Wigglesworthia & 1 \\
Escherichia & 35 & Xenorhabdus & 1 \\
Klebsiella & 4 & Yersinia & 14 \\
Pectobacterium & 3 & Erwinia & 4 \\
Proteus & 3 & Candidatus & 3 \\
\hline
\end{tabular}

* Candidatus is not a genus; however some strains were included as they were classified as Enterobacteriaceae at the time of study.

Twelve bacterial strains included in experimental evaluation of the chip are listed in Table 3 (Results section).

\subsection{Pan-Genomics}

The pan-genome was estimated, as described by Snipen et al [27]. Briefly, all protein sequences were compared by BLASTP [28]. Two proteins were attributed to a single gene family if they satisfied the 50/50 rule, meaning that when they could produce a pairwise BLASTP alignment covering at least 50\% amino of the length of the longest protein with at least $50 \%$ of amino acid identity. Each genome was compared successively: for each $n$ additional genome, that genome was compared to any combinations of $n-1$ genomes and the number of identical 'core genes' and 'genome specific genes' (specific for genome $n$ ) were counted for each $n$. All cumulative BLASTP hits found in the whole set of genomes were plotted as a running total and were considered as pan-genome, which increases as more genomes are added. The number of gene families with at least one representative in every genome was plotted for the core-genome.

\footnotetext{
${ }^{1}$ Available at http://www.cbs.dtu.dk/ dave/Supplementary_TableS1.pdf
} 


\subsection{The Custom-Microarray Design}

The custom probe set for the microarrays was designed around 78 different groups of genomes (the list of groups is presented in the Results section, Table 2) including a collection of generic probes for the entire enteric core (97 genes), as well as for the probes that differentiate each genus within Enterobacteriaceae. The custom probe set was followed by more specialized probe sets for species-specific classification within Klebsiella, Salmonella, Escherichia, Shigella, and Yersinia genera and further probe groups were specific for strain and pathotype for Escherichia coli genus. Additionally, sets of probes for all the gene families, comprising pan-genome, were included. The custom microarrays, manufactured by NimbleGen, were based on the NimbleGen 12-plex platform.

\subsection{Constructing Target Gene Sets}

The genome sequences in this study (Table S1) were searched for genes using the Prodigal gene-finding approach [29] in order to standardize gene finding. All proteincoding sequences were aligned all-against-all using BLASTP [28], and similarity was decided according to 50/50 rule. Proteins that satisfy this rule were assigned to one protein family. 'Group specific gene families' (as described above) were found using batch Perl script, which outputs a list of gene families that are either common to or complementary to the genomes included in pan- and core-genome plots (depending on whether unique or core genes are extracted). Representative sequences from each gene network were selected by choosing the organism from which the genes should be extracted. Unique genes were considered to be those that appeared to be conserved only among the strains belonging to a particular group.

\subsection{Probe Selection for Target Genes}

Probes for target genes were selected using the OligoWiz program, previously described by Wernersson et al. [30][31]. At each position along all the input sequence, the suitability of placing a probe was evaluated according to several criteria: melting temperature $\left(\Delta T_{m}\right)$, cross-hybridization, folding (self-annealing), position (within the transcript), and 'low-complexity' (absence of subsequences that occur very commonly in the genome/transcriptome). The weighting scores for these criteria are as follow: cross-hybridization, $39 \% ; \Delta T_{m}, 26 \%$; folding, $13 \%$; position, $13 \%$; and lowcomplexity, $9 \%$. No probes were accepted unless an overall score of at least 0.3 was obtained, and all probes were required to have a length in the range of $42 \mathrm{bp}$ to $50 \mathrm{bp}$. OligoWiz was originally designed for single genome use, and thus, the program was modified in order to make the mechanisms screening for cross-hybridization less strict as described by Vejborg et al. [32]. A new modified scheme included a logtransformation in the underlying calculations. The net effect is insignificant near the upper boundary of the score, but next to the lower boundary it increases the discriminatory power of the tool.

$$
\text { BLAST max score }=1-\sum_{n}^{i=1} \log \left(1+\sum_{m}^{m=1} \frac{h m, i}{100}\right.
$$




\subsection{Probe Evaluation in silico}

Probes were aligned against a database consisting of all possible gene sequences in the total data set using BLASTN. The affinity of each probe for every gene was determined and expressed as the number of identical base pairs and by the E-value. Sequences for which the E-value was lower than 0 were extracted using a batch Perl script. Probes that matched strains not expected to belong to particular group were excluded from the further analysis. If more than ten probes per gene remained available after filtering, only not-overlapping ones were used for subsequent analysis. This resulted in the reduction of candidate probes from 106,657 to 53,644. Consequently, the number of probes targeting each gene ranged from 3 to 14 with a median coverage of about 7 probes per gene.

\subsection{DNA Preparation and Hybridization}

All the experimental isolates were kindly provided by the laboratory of Frank Møller Aarestrup (DTU Food, The Technical University of Denmark). All test strains were grown overnight on blood agar and genomic DNA was isolated as described in the protocol for the Easy-DNA kit from Invitrogen [33]. The method used is briefly described here: the lysis of the cells was performed by the addition of solution A and subsequent incubation at $65^{\circ} \mathrm{C}$. Proteins and lipids were precipitated and extracted by the addition of solution $\mathrm{B}$ and chloroform. The solution was then centrifuged to separate the solution into two phases. The DNA was in the upper, clear aqueous phase, the proteins and lipids were in the solid interface, and the chloroform formed the lower phase. The DNA was then removed, precipitated with ethanol, and re-suspended in TE buffer.

The genomic DNA was labeled with cy3 dye and hybridized to NimbleGen custom arrays according to Arrays User's Guide for CGH analysis as provided by the manufacturer of the arrays (Roche NimbleGen, Madison, Wisconsin, USA).

\subsection{Analysis Methods}

In the initial step, the raw data from multiple microarrays was extracted using NimbleScan software, developed by Roche NimbleGen, and combined as a single input. Data analysis was performed in R (a statistical software program), using the 'oligo' package for analyzing oligonucleotide arrays at the probe level. The package was obtained from Bioconductor [34]. The probes were mapped to each gene group, including position, according to the design. Chip analysis workflow then continued as follows:

1. Performance of probe-level normalization using robust multi-array average (RMA) algorithm. RMA method had a three-step procedure consisting of background correction, normalization, and summarization to obtain gene-level relative intensity measures from probe-level intensities [35].

2. Estimation of gene 'on/off' status based on the summarized gene relative intensities and the median of these intensities for each of the 78 groups.

Supporting microarray chip design information is publicly available ${ }^{2}$.

\footnotetext{
${ }^{2}$ http://www.cbs.dtu.dk/ dave/Microarray_Chip_Design_Lukjancenko_2010.ndf
} 


\section{Results}

\subsection{Pan-Genome and Core-Genome Estimation}

For each of the considered bacterial strains listed in Table S1 (Supplementary data), the genome sequence was downloaded from NCBI/GenBank. Genes were predicted by Prodigal [29], and translated into proteins. This resulted in a dataset of 887,184 entries with considerable redundancy due to the presence of the same gene in multiple genomes. To reduce the homology, proteins were grouped into the gene families. Proteins were considered conserved (belonging to the same gene group) if they showed at least $50 \%$ amino acid identity in a BLASTP alignment covering at least $50 \%$ of the length of the longest protein. The combined pan-genome of 116 genomes within Enterobacteriaceae was estimated and appeared to contain 44,838 gene families. The core-genome, that is, the number of conserved genes present in all 116 genomes, was estimated to be comprised of 97 conserved gene families.

\subsection{Probe and Microarray Design}

In the presented Enterobacteriaceae pan-genome microarray design strategy, the probe set was designed around 78 different groups of genomes. The microarray was made up of a collection of probes for each genus within Enterobacteriaceae, being species-specific for Klebsiella, Salmonella, Escherichia, Shigella, and Yersinia genera; strain and pathotype specific for Escherichia coli genus; core genes; and all protein families, comprising pan-genome. Using the data from the pan- and core-genome estimation step, the number of 'group-specific' genes and probes was determined and are shown in Table 2. Genes were considered to be 'group-unique' if they were found only within genomes, belonging to a particular group, and were absent in all of the rest genomes among a set of 116 genomes.

The final result was a set of 52,356 Enterobacteriaceae target sequences, representing genes of both specific groups and pan-genome gene families. The oligos were then selected using OligoWiz [31] based on several criteria, including their specificity, self-annealing, presence of low-complexity sequences, and their lengths adjusted so as to standardize the hybridization strength. Probes were filtered in order to avoid complimentarity with unwanted targets. In the end a set of 130,540 non-overlapping probes with an average length of $49 \mathrm{bp}$ were obtained. The average number of probes per target gene was about 7, although the actual number for any given target depended on the length of the sequence, since shorter sequences have space for fewer nonoverlapping probes. For set of probes that represent gene families an average of 3 probes per family was used.

\subsection{Validation of the Custom Arrays}

The chip design was evaluated by analyzing and comparing hybridization data from twelve control strains, shown in Table 3. Microarray data can have noise, coming from multiple variations which can occur during the array manufacturing process, the preparation of the biological sample for the hybridization, the hybridization of the samples to the array itself, and the quantification of the spot intensities [35]. To remove such variation, which obviously will affect the measured gene intensity levels, 
Table 2. Number of 'group specific' gene families and probes before and after in silico validation

\begin{tabular}{|c|c|c|c|c|}
\hline Probe group & $\begin{array}{c}\text { Number } \\
\text { of genes } \\
\text { before } \\
\text { validation }\end{array}$ & $\begin{array}{c}\text { Number } \\
\text { of probes } \\
\text { before } \\
\text { validation }\end{array}$ & $\begin{array}{c}\text { Number } \\
\text { of genes } \\
\text { after } \\
\text { validation }\end{array}$ & $\begin{array}{c}\text { Number } \\
\text { of probes } \\
\text { after } \\
\text { validation }\end{array}$ \\
\hline Buchnera genus & 14 & 200 & 14 & 123 \\
\hline Candidatus strains & 41 & 584 & 41 & 373 \\
\hline Citrobacter genus & 20 & 171 & 15 & 95 \\
\hline Cronobacter genus & 271 & 3224 & 270 & 2002 \\
\hline Dickeya genus & 155 & 2129 & 155 & 1398 \\
\hline Edwardsiella genus & 318 & 3803 & 317 & 2447 \\
\hline Enterobacter genus & 40 & 511 & 40 & 318 \\
\hline Erwinia genus & 217 & 2919 & 217 & 1840 \\
\hline Escherichia genus & 1 & 15 & 1 & 10 \\
\hline Escherichia coli 042 & 106 & 1047 & 79 & 450 \\
\hline Escherichia coli 536 & 142 & 1207 & 95 & 436 \\
\hline Escherichia coli 55989 & 72 & 646 & 45 & 272 \\
\hline Escherichia coli APEC & 116 & 1287 & 14 & 83 \\
\hline Escherichia coli APEC O1 & 116 & 1287 & 14 & 83 \\
\hline Escherichia coli Avirulent & 69 & 508 & 39 & 241 \\
\hline Escherichia coli B phylogroup & 14 & 175 & 14 & 100 \\
\hline Escherichia coli CFT073 & 292 & 2251 & 115 & 393 \\
\hline Escherichia coli $\mathrm{E} 24377 \mathrm{~A}$ & 249 & 1700 & 90 & 511 \\
\hline Escherichia coli EAEC & 72 & 646 & 45 & 272 \\
\hline Escherichia coli ED1a & 159 & 1545 & 146 & 823 \\
\hline Escherichia coli $\mathrm{EHEC}$ & 21 & 173 & 13 & 27 \\
\hline Escherichia coli EPEC & 142 & 1685 & 126 & 893 \\
\hline Escherichia coli ETEC & 249 & 1700 & 90 & 511 \\
\hline Escherichia coli ExPEC & 52 & 392 & 17 & 131 \\
\hline Escherichia coli $\mathrm{HS}$ & 90 & 642 & 44 & 313 \\
\hline Escherichia coli IAI1 & 67 & 499 & 39 & 238 \\
\hline Escherichia coli IAI39 & 77 & 609 & 48 & 262 \\
\hline Escherichia coli $\mathrm{K}-12$ & 11 & 159 & 11 & 113 \\
\hline Escherichia coli $\mathrm{O} 103: \mathrm{H} 2$ & 65 & 693 & 50 & 377 \\
\hline Escherichia coli $\mathrm{O} 111: \mathrm{H}-$ & 148 & 1536 & 54 & 250 \\
\hline Escherichia coli $\mathrm{O} 127: \mathrm{H} 6$ & 142 & 1685 & 126 & 893 \\
\hline Escherichia coli $\mathrm{O} 157: \mathrm{H} 7$ & 68 & 709 & 52 & 379 \\
\hline Escherichia coli $\mathrm{O} 26: \mathrm{H} 11$ & 74 & 690 & 48 & 280 \\
\hline Escherichia coli $\mathrm{S} 88$ & 52 & 392 & 17 & 131 \\
\hline Escherichia coli SE11 & 178 & 1692 & 70 & 360 \\
\hline Escherichia coli SE15 & 58 & 609 & 49 & 328 \\
\hline Escherichia coli SMS-3-5 & 145 & 1064 & 106 & 501 \\
\hline Escherichia coli UMN026 & 113 & 1026 & 85 & 505 \\
\hline Escherichia coli UPEC & 121 & 983 & 49 & 179 \\
\hline Escherichia coli UTI89 & 85 & 754 & 35 & 192 \\
\hline Escherichia/Shigella genera & 15 & 184 & 15 & 113 \\
\hline Klebsiella genus & 242 & 3296 & 242 & 2090 \\
\hline Klebsiella pneumoniae 342 & 11 & 93 & 8 & 50 \\
\hline Klebsiella pneumoniae MGH 78578 & 21 & 237 & 14 & 49 \\
\hline Klebsiella pneumoniae NTUH-K2044 & 339 & 2636 & 233 & 863 \\
\hline
\end{tabular}


Table 2. (Continued)

\begin{tabular}{|c|c|c|c|c|}
\hline Klebsiella variicola At-22 & 115 & 1282 & 110 & 758 \\
\hline Pectobacterium genus & 166 & 2287 & 166 & 1422 \\
\hline Proteus genus & 355 & 4782 & 355 & 3006 \\
\hline Photorhadbus genus & 318 & 4392 & 318 & 2728 \\
\hline Salmonella genus & 69 & 933 & 69 & 575 \\
\hline Salmonella enterica Agona & 136 & 1151 & 111 & 568 \\
\hline Salmonella arizonae & 477 & 3828 & 474 & 2245 \\
\hline Salmonella enterica Choleraesuis & 92 & 804 & 44 & 87 \\
\hline Salmonella enterica Dublin & 101 & 526 & 22 & 77 \\
\hline Salmonella enterica Enteritidis & 20 & 217 & 9 & 55 \\
\hline Salmonella enterica Gallinarum & 10 & 88 & 5 & 14 \\
\hline Salmonella enterica Heidelberg & 91 & 608 & 51 & 249 \\
\hline Salmonella enterica Newport & 189 & 1967 & 111 & 351 \\
\hline Salmonella enterica Paratyphi A & 10 & 80 & 7 & 10 \\
\hline Salmonella enterica Paratyphi B & 436 & 1982 & 175 & 547 \\
\hline Salmonella enterica Paratyphi $C$ & 54 & 266 & 20 & 47 \\
\hline Salmonella enterica Schwarzengrund & 139 & 1025 & 122 & 498 \\
\hline Salmonella enterica Typhi & 69 & 759 & 63 & 326 \\
\hline Salmonella enterica Typhimurium & 9 & 113 & 3 & 30 \\
\hline Serratia genus & 780 & 10393 & 780 & 6777 \\
\hline Shigella boydii & 19 & 164 & 16 & 52 \\
\hline Shigella dysenteriae & 113 & 1216 & 98 & 348 \\
\hline Shigella flexneri & 17 & 218 & 17 & 123 \\
\hline Shigella genus & 28 & 401 & 25 & 178 \\
\hline Shigella sonnei & 48 & 531 & 32 & 152 \\
\hline Sodalis genus & 420 & 5697 & 420 & 3464 \\
\hline Wigglesworthia genus & 212 & 3029 & 212 & 1789 \\
\hline Xenorhabdus genus & 82 & 855 & 82 & 527 \\
\hline Yersinia genus & 97 & 4189 & 97 & 809 \\
\hline Yersinia enterocolitica & 336 & 1312 & 336 & 2655 \\
\hline Yersinia pestis & 7 & 26 & 5 & 5 \\
\hline Yersinia pseudotuberculosis & 23 & 165 & 13 & 24 \\
\hline Core genes & 97 & 1378 & 97 & 850 \\
\hline Gene families & 42151 & 180219 & 27536 & 76896 \\
\hline
\end{tabular}

normalization was performed. A set of twelve arrays (one 12plex array) used in the experiment was printed at the same time, so background noise effects were expected to be reasonably similar across all arrays. Only one out of the twelve the results were not as anticipated. The single exception being for the Salmonella enterica serovar Choleraesuis isolate, which shows variation. Thus it was decided to exclude hybridization data of this isolate from further analysis. RMA normalization, performed for microarray data of the remaining eleven samples, made the distribution of probe intensities for each array in a set of arrays nearly the same.

In the workflow of further microarray data analysis, the evaluation of which genus, species, pathotype/serovar or strain, the experimental isolate is most likely to be similar to. For each of the seventy-eight gene sets, the median of signal intensities were calculated. The analysis was performed based on both distribution of probe log intensities and the signal median. The examples are shown in Figures 1-3, which visualize 


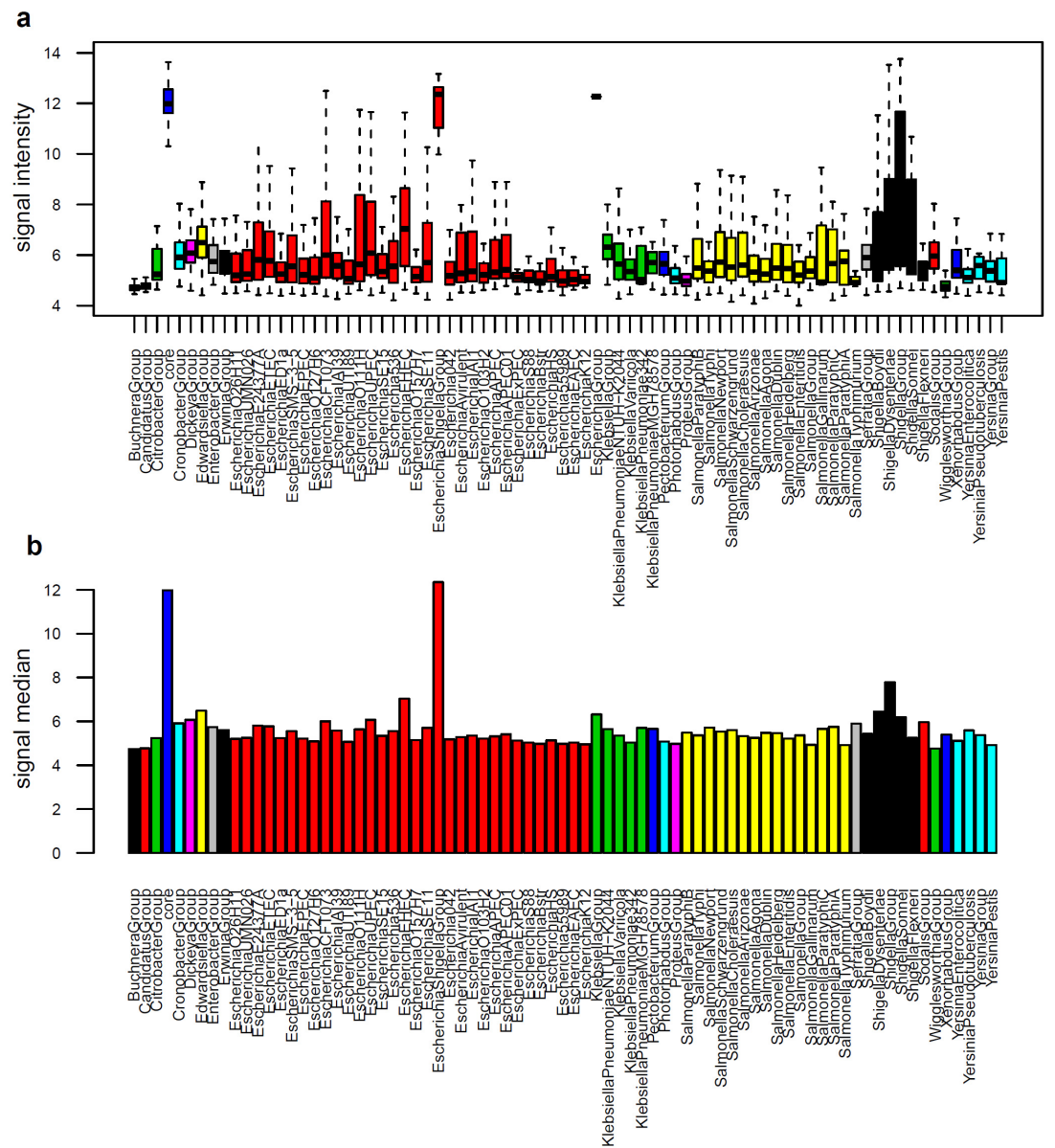

Fig. 1. Distribution of signal intensity and signal median for Escherichia coli ECOR20 strain among the set of seventy-eight groups, mentioned previously in Table 2. a. Box-and-whisker plot, showing signal intensity distribution. $b$. Bar plot, showing expression signal median distribution. X-axis elements are sorted by genus, based on the order showed in Table 2. Colour code is based on the genera, where 12-colour palette represents 20 genera.

the resulting plots for single representative of three chosen genera Escherichia, Salmonella and Yersinia. Those were Escherichia coli ECOR20, Salmonella enterica serovar Dublin and Yersinia frederiksii, respectively. Table 3 overviews the results for all the eleven isolates, used in the study.

Both box-and-whisker and bar plots for Escherichia coli ECOR20, represented in Fig. 1, show high signal intensity among the genes comprising core and Escherichiaand-Shigella groups. Additionally, results show high similarity to several pathogenic E. coli strains, such as Escherichia coli CFT073, and strains of O111:H-, UPEC and EHEC pathotypes. Apart from being highly expressed among the genes belonging to Escherichia genus, microarray data show relatively high signal level to Shigella genus 

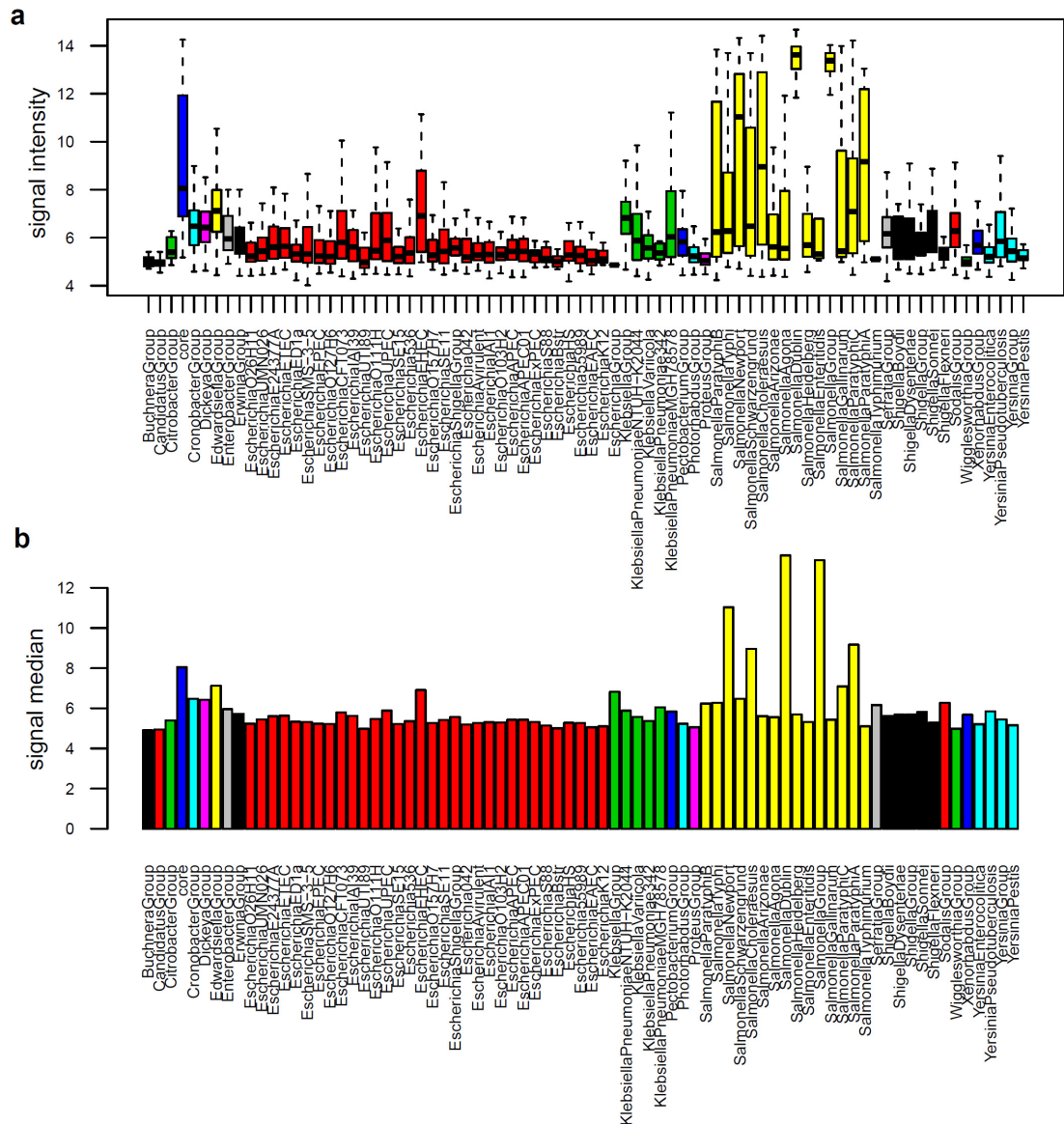

Fig. 2. Distribution of signal intensity and signal median for Salmonella enterica serovar Dublin strain among the set of seventy-eight groups, mentioned previously in Table 2. a. Box-andwhisker plot, showing signal intensity distribution. b. Bar plot, showing expression signal median distribution. $\mathrm{X}$-axis elements are sorted by genus, based on the order showed in Table 2. Colour code is based on the genera, where 12-colour palette represents 20 genera.

strains, thus, resulting in another proof of Escherichia and Shigella genera strains being very similar.

Fig. 2 visualizes the comparison of data for Salmonella enterica serovar Dublin isolate. Genes have high intensity values within strains belonging to Salmonella genus and core group. The highest similarity is shown to be Dublin serovar; however, DNA sequences appeared to hybridize with the high strength to Newport, Choleraesuis and Paratyphi A serovar representing probes as well.

In the case of the chosen representative for Yersinia genus, Yersinia frederiksi, results, shown in Fig. 3, are not that positive, since any obvious high intensity signal cannot be seen. This might occur as a consequence of impropriate isolation of genomic DNA, low concentration of labeled DNA, which was obviously not enough for proper hybridization to target genes, or cross-hybridization effect. 
a
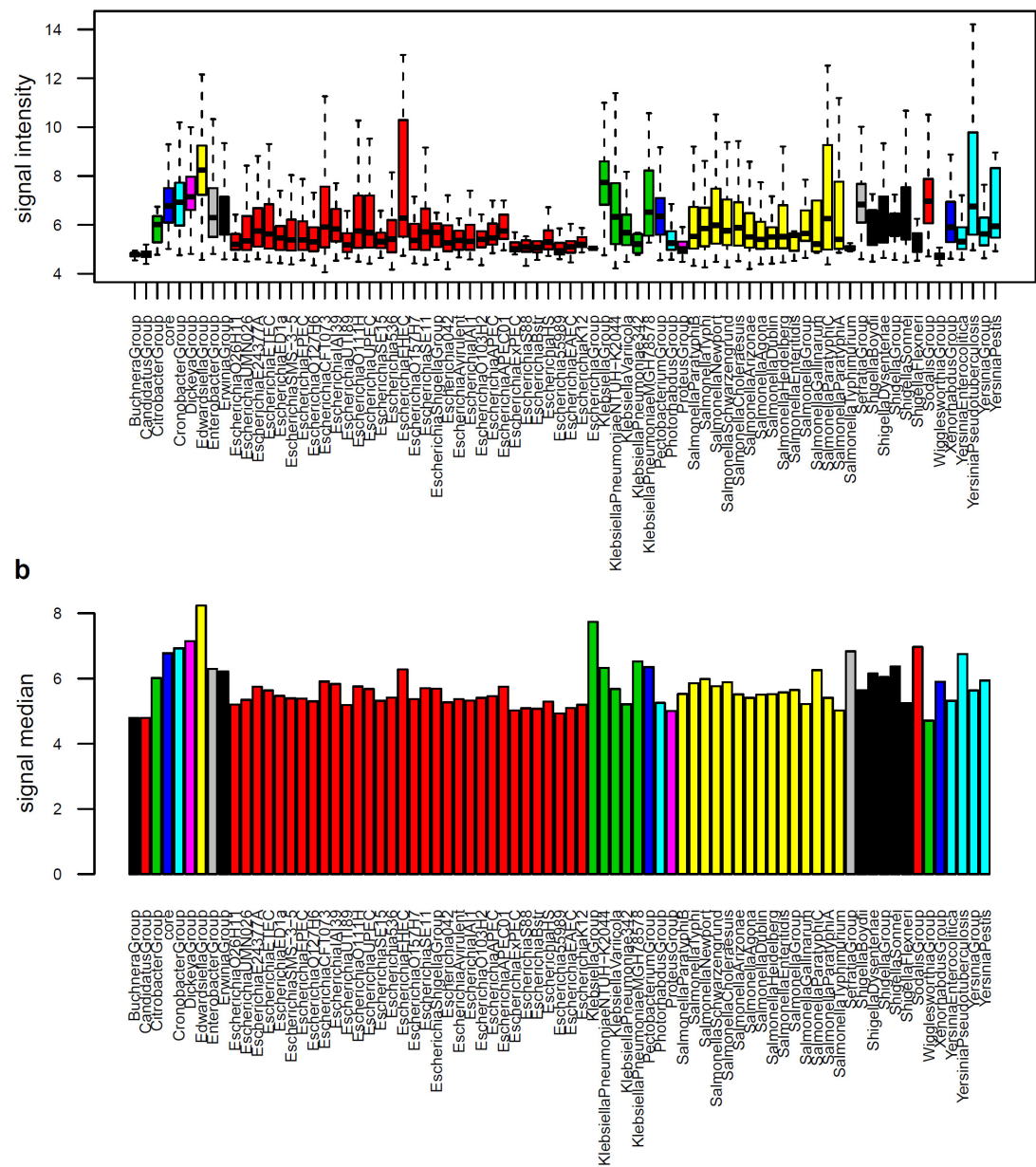

Fig. 3. Distribution of signal intensity and signal median for Yersinia frederiksii strain among the set of seventy-eight groups, mentioned previously in Table 2. a. Box-and-whisker plot, showing signal intensity distribution. b. Bar plot, showing expression signal median distribution. X-axis elements are sorted by genus, based on the order showed in Table 2. Colour code is based on the genera, where 12-colour palette represents 20 genera.

Isolates, results for which are presented in Table 3, show different chip performances. Several of them can be easily proved to belong to a particular genus, specific species and be most likely similar to a particular genus, species or serovar/serotype.

However, some samples, likewise Yersinia frederiksii, do not show obvious results. This can consider the presence of uncertainties included in genomic DNA purification and sample preparation for the hybridization. 
Table 3. Overview of experimental validation results

\begin{tabular}{lccl}
\hline Isolate / Distinguishing level & Genera & Species & Pathotype/Serovar \\
\hline Escherichia coli ECOR20 & + & + & - \\
Salmonella enterica serovar Dublin D6 & + & + & + \\
Salmonella enterica serovar Paratyphi B var Java b & + & + & + \\
Salmonella enterica serovar Isangi 2005-60-2087-1 & + & + & \\
Salmonella enterica Typhimurium HN-GSS-2007-016 & + & + & + \\
Salmonella enterica serovar Choleraesuis 2870/08 & & & \\
Shigella sonnei phase 12006-077 & - & - & \\
Shigella flexneri 4 2006-054 & + & + & \\
Shigella boydii 9S & - & - & - \\
Yersinia entericolitica O3 98-30624-5 & - & - & - \\
Yersinia ruckerii NCTC 10476 & - & - & - \\
Yersinia frederiksii P963 & - & - & - \\
'+' is a positive result, '-' is a negative result and absence of any mark means no analysis with \\
this purpose was made or results are not analysed
\end{tabular}

\section{Discussion and Perspective}

The design of a microarray chip covering 116 bacterial genomes has proven to be a considerable challenge. Multiple aspects had to be examined, such as the number of possible sequences to be included in the database, various criteria to select the unique set of genes to particular groups of genomes, and to design probes for them. The greatest difficulty was to optimize these criteria and to filter out the false positive representative sequences for each sequence of interest. Some genera within Enterobacteriaceae, such as Escherichia and Shigella, are quite similar, thus it was difficult to find genus-specific genes. For example, the Escherichia genus appeared to have only a single gene family conserved among all the strains belonging to this genus, and being absent in the other enterics. Thus it was an obvious decision to design probes for Escherichia-and-Shigella genera-specific genes.

Along with choosing representative sequence for each of unique gene family, a problem of selecting the right organism to extract representative sequences for coregenome set became evident. In this study, core-genome genes were extracted from type species of the type genus Escherichia coli K-12 MG1655 strain. The unique sets of genes were selected on protein level, that is, similarity/dissimilarity was based on alignment using BLASTP, and gene family members were considered based on the $50 / 50$ rule, described above. Thus this might be an explanation of why some probes did not show high intensity levels at the DNA level as was predicted.

Selecting the probes is indeed a challenging aspect. On the one hand, probes should cover all versions of the same gene, however, at the same time they should be able to distinguish between different genera, species, pathotypes/serovars, and strains. Furthermore, the array should allow various numbers of probes per gene in order to acquire the sufficient coverage of genes. Longer sequences require higher numbers of probes, whereas design of the same number of probes for short genes would result in low quality probes [36]. Therefore, the challenge is to find the best possible solution, with least time, money, and personal energy consumption. 
Several improvements and suggestions could be considered for the design of an Enterobacteriaceae pan-genome microarray chip. To obtain more sufficient unique gene finding, searchs should be done on DNA level with an appropriate cut-off value. Alignment using the BLASTN algorithm would be able to efficiently identify homologous nucleotide sequences based on similarity and would be helpful in avoiding non-specific probes.

Furthermore, for the validation of the chip step, sample preparations, such as genomic DNA isolation, labeling, and preparation to hybridize an array should be done according to protocols. Purity of DNA should be checked before the DNA labeling step to avoid small quantities of labeled DNA, which hybridizes to wrong sequences and fails to recognize the expected target sequence.

\section{Conclusion}

In this study, an Enterobacteriaceae pan-genome microarray chip was developed based on 116 genomes within this bacterial family. The typical genome size (with the exception of the reduced endosymbiont genomes of Buchnera, Wigglesworthia and Sodalis genera) contained between 3500 and 5500 genes. This made it possible to find at least 10 genus-, species- and pathotype/serovar-genes among all the analysed genomes. This resulted in 53644 unique probes, which were expected to hybridize to particular target sequence. High-density pan-genome microarrays can be very useful in both characterizing DNA content and monitoring expression levels for thousands of genes simultaneously. The comparison of two or more arrays can display the distinct patterns of gene expression or signal intensity level that are useful in the definition of unknown strains or genes included in these genomes. Using some experimental tests the ability of the microarray to determine bacterial strains within Escherichia spp., Shigella spp., Salmonella spp. and Yersinia spp. was demonstrated. Most of the results showed discriminative power, although some samples did not show a clear connection to the bacterial strain they are most likely to be similar to. This could be due to low quality DNA from the experiment.

It can be concluded that a Enterobacteriaceae pan-genome microarray, based on 116 genomes provides a perfect tool for determination of the genetic makeup of unknown strains within this bacterial family and can introduce insights into phylogenetic relationships.

Acknowledgments. This work is supported by grants from the Danish Center for Scientific Computing and the Danish Research Council. The authors would like to thank Colleen Ussery for help in editing the manuscript.

\section{References}

1. Hall, B.G., Ehrlich, G.D., Hu, F.Z.: Pan-genome analysis provides much higher strain typing resolution than multi-locus sequence typing. Microbiology 156, 1060-1068 (2010)

2. Sørensen, T.I., Nielsen, G.G., Andersen, P.K., Teasdale, T.W.: Genetic and environmental influences on premature death in adult adoptees. N. Engl. J. Med. 318, 727-732 (1988) 
3. Helms, M., Vastrup, P., Gerner-Smidt, P., Mølbak, K.: Short and long term mortality associated with foodborne bacterial gastrointestinal infections: registry based study. BMJ 326, 357 (2003)

4. Ternhag, A., Törner, A., Svensson, A., Ekdahl, K., Giesecke, J.: Short- and long-term effects of bacterial gastrointestinal infections. Emerging Infect. Dis. 14, 143-148 (2008)

5. Mead, P.S., Slutsker, L., Dietz, V., McCaig, L.F., Bresee, J.S., Shapiro, C., Griffin, P.M., Tauxe, R.V.: Food-related illness and death in the United States. Emerging Infect. Dis. 5, 607-625 (1999)

6. Litrup, E., Torpdahl, M., Malorny, B., Huehn, S., Helms, M., Christensen, H., Nielsen, E.M.: DNA microarray analysis of Salmonella serotype Typhimurium strains causing different symptoms of disease. BMC Microbiol. 10, 96 (2010)

7. Laupland, K.B., Schønheyder, H.C., Kennedy, K.J., Lyytikäinen, O., Valiquette, L., Galbraith, J., Collignon, P.: Salmonella enterica bacteraemia: a multi-national populationbased cohort study. BMC Infect. Dis. 10, 95 (2010)

8. Cheng, S., Hu, Y., Zhang, M., Sun, L.: Analysis of the vaccine potential of a natural avirulent Edwardsiella tarda isolate. Vaccine 28, 2716-2721 (2010)

9. Lindberg, A.M., Ljungh, A., Ahrné, S., Löfdahl, S., Molin, G.: Enterobacteriaceae found in high numbers in fish, minced meat and pasteurised milk or cream and the presence of toxin encoding genes. Int. J. Food Microbiol. 39, 11-17 (1998)

10. Musgrove, M.T., Northcutt, J.K., Jones, D.R., Cox, N.A., Harrison, M.A.: Enterobacteriaceae and related organisms isolated from shell eggs collected during commercial processing. Poult. Sci. 87, 1211-1218 (2008)

11. Stiles, M.E., Ng, L.K.: Enterobacteriaceae associated with meats and meat handling. Appl. Environ. Microbiol. 41, 867-872 (1981)

12. Wright, C., Kominos, S.D., Yee, R.B.: Enterobacteriaceae and Pseudomonas aeruginosa recovered from vegetable salads. Appl. Environ. Microbiol. 31, 453-454 (1976)

13. Cossart, P., Sansonetti, P.J.: Bacterial invasion: the paradigms of enteroinvasive pathogens. Science 304, 242-248 (2004)

14. Hornef, M.W., Wick, M.J., Rhen, M., Normark, S.: Bacterial strategies for overcoming host innate and adaptive immune responses. Nat. Immunol. 3, 1033-1040 (2002)

15. Olsson, C., Ahrné, S., Pettersson, B., Molin, G.: DNA based classification of food associated Enterobacteriaceae previously identified by biology microplates. Syst. Appl. Microbiol. 27, 219-228 (2004)

16. Glasner, J.D., Marquez-Villavicencio, M., Kim, H., Jahn, C.E., Ma, B., Biehl, B.S., Rissman, A.I., Mole, B., Yi, X., Yang, C., Dangl, J.L., Grant, S.R., Perna, N.T., Charkowski, A.O.: Niche-specificity and the variable fraction of the Pectobacterium pan-genome. Mol. Plant Microbe Interact 21, 1549-1560 (2008)

17. Tettelin, H., et al.: Genome analysis of multiple pathogenic isolates of Streptococcus agalactiae: implications for the microbial "pan-genome". Proc. Natl. Acad. Sci. U.S.A. 102, 13950-13955 (2005)

18. Lefébure, T., Stanhope, M.J.: Evolution of the core and pan-genome of Streptococcus: positive selection, recombination, and genome composition. Genome Biol. 8, 71 (2007)

19. Phillippy, A.M., Deng, X., Zhang, W., Salzberg, S.L.: Efficient oligonucleotide probe selection for pan-genomic tiling arrays. BMC Bioinformatics 10, 293 (2009)

20. Pinkel, D., Segraves, R., Sudar, D., Clark, S., Poole, I., Kowbel, D., Collins, C., Kuo, W.L., Chen, C., Zhai, Y., Dairkee, S.H., Ljung, B.M., Gray, J.W., Albertson, D.G.: High resolution analysis of DNA copy number variation using comparative genomic hybridization to microarrays. Nat. Genet. 20, 207-211 (1998) 
21. Wang, D.G., Fan, J.B., Siao, C.J., Berno, A., Young, P., Sapolsky, R., Ghandour, G., Perkins, N., Winchester, E., Spencer, J., Kruglyak, L., Stein, L., Hsie, L., Topaloglou, T., Hubbell, E., Robinson, E., Mittmann, M., Morris, M.S., Shen, N., Kilburn, D., Rioux, J., Nusbaum, C., Rozen, S., Hudson, T.J., Lipshutz, R., Chee, M., Lander, E.S.: Large-scale identification, mapping, and genotyping of single-nucleotide polymorphisms in the human genome. Science 280, 1077-1082 (1998)

22. Khodursky, A.B., Peter, B.J., Cozzarelli, N.R., Botstein, D., Brown, P.O., Yanofsky, C.: DNA microarray analysis of gene expression in response to physiological and genetic changes that affect tryptophan metabolism in Escherichia coli. Proc. Natl. Acad. Sci. U.S.A. 97, 12170-12175 (2000)

23. Wei, Y., Lee, J.M., Richmond, C., Blattner, F.R., Rafalski, J.A., LaRossa, R.A.: Highdensity microarray-mediated gene expression profiling of Escherichia coli. J. Bacteriol. 183, 545-556 (2001)

24. Jacobsen, L., Durso, L., Conway, T., Nickerson, K.W.: Escherichia coli O157:H7 and other E. coli strains share physiological properties associated with intestinal colonization. Appl. Environ. Microbiol. 75, 4633-4635 (2009)

25. Willenbrock, H., Fridlyand, J.: A comparison study: applying segmentation to array cgh data for downstream analyses. Bioinformatics 21, 4084-4091 (2005)

26. Willenbrock, H., Petersen, A., Sekse, C., Kiil, K., Wasteson, Y., Ussery, D.W.: Design of a seven-genome Escherichia coli microarray for comparative genomic profiling. J. Bacteriol. 188, 7713-7721 (2006)

27. Snipen, L., Almøy, T., Ussery, D.W.: Microbial comparative pan-genomics using binomial mixture models. BMC Genomics 10, 385 (2009)

28. Altschul, S.F., Madden, T.L., Schäffer, A.A., Zhang, J., Zhang, Z., Miller, W., Lipman, D.J.: Gapped BLAST and psi-BLAST: a new generation of protein database search programs. Nucleic. Acids. Res. 25, 3389-3402 (1997)

29. Hyatt, D., Chen, G., Locascio, P.F., Land, M.L., Larimer, F.W., Hauser, L.J.: Prodigal: prokaryotic gene recognition and translation initiation site identification. BMC Bioinformatics 11,119 (2010)

30. Wernersson, R., Nielsen, H.B.: OligoWiz 2.0-integrating sequence feature annotation into the design of microarray probes. Nucleic Acids Res. 33, W611-W615 (2005)

31. Wernersson, R., Juncker, A.S., Nielsen, H.B.: Probe selection for DNA microarrays using OligoWiz. Nat. Protoc. 2, 2677-2691 (2007)

32. Vejborg, R.M., Bernbom, N., Gram, L., Klemm, P.: Anti-adhesive properties of fish tropomyosins. J. Appl. Microbiol. 105, 141-150 (2008)

33. Easy-DNA kit (2010),

http://tools.invitrogen.com/content/sfs/manuals/easydna_man.pdf

34. Gentleman, R.C., Carey, V.J., Bates, D.M., Bolstad, B., Dettling, M., Dudoit, S., Ellis, B., Gautier, L., Ge, Y., Gentry, J., Hornik, K., Hothorn, T., Huber, W., Iacus, S., Irizarry, R., Leisch, F., Li, C., Maechler, M., Rossini, A.J., Sawitzki, G., Smith, C., Smyth, G., Tierney, L., Yang, J.Y.H., Zhang, J.: Bioconductor: open software development for computational biology and bioinformatics. Genome Biol. 5, R80 (2004)

35. Do, J.H., Choi, D.: Normalization of microarray data: single-labeled and dual-labeled arrays. Mol. Cells 22, 254-261 (2006)

36. Willenbrock, H., Hallin, P.F., Wassenaar, T.M., Ussery, D.W.: Characterization of probiotic Escherichia coli isolates with a novel pan-genome microarray. Genome Biol. 8, 267 (2007) 